\title{
CARACTERIZACIÓN DE UNA ESPIROLACTONA SESQUITERPÉNICA $\alpha$-METILÉNICA OBTENIDA DE Ambrosia arborescens Miller Y EVALUACIÓN DE SU ACTIVIDAD BIOLÓGICA EN Tripanosoma cruzi
}

\author{
Teresa Cano de Terrones*
}

\begin{abstract}
RESUMEN
Ambrosia arborescens Miller, denominada comúnmente "marco"; es una planta aromática, medicinal que se encuentra en América del Sur, mayormente en los Andes del Perú, en el Departamento de Arequipa. El estudio de los constituyentes químicos de la parte aérea de Ambrosia arborescens permitió obtener una espirolactona sesquiterpénica. Para su separación y aislamiento se utilizó métodos cromatográficos de columna, capa fina y cromatografía líquida de alta eficacia. La elucidación estructural se realizó a través de métodos espectroscópicos: resonancia magnética nuclear protónica $(\mathrm{RMN}-1 \mathrm{H})$, del carbono-13 (RMN-13C), espectroscopía infrarroja (IR) y espectrometría de masas (EM). En la determinación de la estereoquímica y las conformaciones se empleó experimentos bidimensionales de espectroscopía: COSY (Correlation Spectroscopy), HSQC (Heteronuclear Single Quantum Correlation), HMBC (Heteronuclear Multiple Bond Correlation) y ROESY (Rotational nuclear Overhouser Effect Spectroscopy). Desde un punto de vista teórico, el análisis conformacional fue realizado a través de Mecánica Molecular empleando el programa MM2 de Hyperchem. La evaluación de la actividad antiparasitaria, in vitro, sobre Tripanosoma cruzi, mostró que a muy bajas concentraciones, el producto obtenido presenta actividad tripanocida.

Palabras clave: Ambrosia arborescens Miller, espirolactona sesquiterpénica $\alpha$ metilénica, actividad tripanocida, Tripanosoma cruzi, elucidación estructural, cromatografía líquida de alta eficacia.
\end{abstract}

\section{CARACTERIZATION OF A SPIRONOLACTONE SESQUITERPENE $\alpha$-METILENICA FROM Ambrosia arborescens Miller AND EVALUATION OF THEIR BIOLOGICAL ACTIVITY IN Tripanosoma cruzi}

\begin{abstract}
Ambrosia arborescens Miller, commonly known as "marco" is a South American aromatic plant found mainly in the Department of Arequipa at the Peruvian Andes. The study of the chemical constituents of Ambrosia arborescens, aerial portion, allowed obtaining a spironolactone sesquiterpene. For its separation and isolation I use chromatographic methods of column, cap it dies and Chromatography liquidates of high efficiency. Structural elucidation was done with spectroscopic methods: protonic nuclear magnetic resonance,
\end{abstract}

Unidad de Posgrado de la Facultad de Ciencias Naturales y Formales, Universidad Nacional de San Agustín, Av. Independencia s/n, Arequipa, Perú, E-mail: tcanefa@gmail.com 
(1H-NMR), carbon-13 (13C-NMR), infrared spectroscopy (IR) and mass spectrometry (MS). To determine stereochemistry and conformations two-dimensional spectroscopic experiments were: COSY (Correlation Spectroscopy), HSQC (Heteronuclear Single Quantum Correlation), HMBC (Heteronuclear Multiple Bond Correlation) and ROESY (Rotational nuclear Overhouser Effect Spectroscopy). From a theoretical viewpoint, conformational analysis was done using the molecular mechanics Hyperchem MM2 program. In vitro antiparisitic activity evaluation on Trypanosoma cruzi showed that, at low concentrations, all products present tripanocide activity.

Key words: Ambrosia arborescens Miller, spironolactone sesquiterpene $\alpha$-metilénica, trypanocidal activity, Trypanosoma cruzi, structural elucidation, chromatography.

\section{INTRODUCCIÓN}

Ambrosia arborescens Miller es una planta aromática medicinal que se encuentra en América del Sur mayormente en los Andes del Perú; se le conoce con los nombres de "marco", "marko", "markhu", "marcju”, "marcu" "marcco", “maleo", “ajenjo", “altamisa", "artemisia", "amargo", "qantin", "jatin".

Es un arbusto de 1,5 a 3 m de altura, rústico verde y poco lignificado, densamente cubierto de pubescencia sedosa plateada. Hojas alternas pinantisectas lanceoladas de 10 a $24 \mathrm{~cm}$ de largo por 7 a $20 \mathrm{~cm}$ de ancho; haz glabescentre, envés densamente albescentre, inflorescencias en densas panojas de color amarillo de 15 a $30 \mathrm{~cm}$ de longitud, racimos de capítulos unisexuales monoicos. Fruto: aquenio de forma ovoidea, rodeado por cuatro prominencias en punta, mide $3 \mathrm{~mm}$ altura y $6 \mathrm{~mm}$ de diámetro. Se le encuentra bajo la forma de matorrales en los bordes de los caminos, cerca de las riberas de los ríos y fuentes de agua, bordeando cultivos, huertos y canales de regadío. Se adapta a diferentes tipos de suelos, soporta helada y sequías, crece desde los 2000 hasta $3500 \mathrm{~m}$ de altitud. ${ }^{2}$ Se le encuentra en el departamento de Arequipa en la Campiña aledaña a la ciudad: Characato, Chiguata, etc; crece en los bordes de los campos, condición natural que precisamente origina que comúnmente se le denomine "marco".

Los cronistas españoles hacen referencia a este recurso señalando que los aborígenes siempre la llevaban consigo dándole muchas aplicaciones para la cura de sus males. La utilizaban para embalsamar cadáveres. ${ }^{3}$

Usaban las hojas soasadas en cocimiento o ungüento graso como antiinflamatorio y antirreumático, en el tratamiento de calambres y aire, el jugo de las ramas para el tratamiento de las hemorroides, para dolores de estómago, para desinflamar los pies para el arrebato y como antiséptico. En los pueblos y caseríos aledaños a la sierra del Perú toda la planta es utilizada (hojas, tallos, raíces, semillas y flores), para el alivio de numerosas enfermedades. Se le utiliza como antitusígeno, antidiarreico y carminativo, para curar la tos bronquitis y asma; además, para preparar insecticidas, fumigaciones o sahumerios. Sus hojas secas y molidas se dejan macerar en agua para usarlas como insecticida. ${ }^{3}$

En la región Arequipa y en otras regiones del Perú se ha encontrado otras especies del género Ambrosia, cuyos estudios etnobotánicos las reportan como medicinales: Ambrosia fruticosa Philippi, Ambrosia sp., Ambrosia peruviana Willd. ${ }^{1,3}$

En esta la región Arequipa, encontramos una gran diversidad de especies vegetales nativas silvestres, ricas en metabolitos secundarios de gran potencial económico dado que dichos recursos podrían aplicarse en la industria alimentaria y farmacéutica.

La mayoría de estas especies son plantas aromáticas y medicinales poco estudiadas en nuestro medio., son consideradas como hierbas malas y por lo tanto, el cultivo de la mayoría de ellas, no se ha sistematizado. 


\section{PARTE EXPERIMENTAL}

Se colectó $30 \mathrm{~kg}$ de partes aéreas de Ambrosia arborescens Miller en el distrito de Chiguata provincia de Arequipa departamento de Arequipa; el lugar de muestreo está situado a $2972 \mathrm{~m}$ sobre el nivel del mar a $16^{\circ} 23^{\prime} 58.9^{\prime \prime}$ de latitud Sur y $71^{\circ} 23$ '29.3” de latitud Oeste.

\section{Extracción $^{4}$}

Se pesó $750 \mathrm{~g}$ de hojas seleccionadas secas y molidas previamente estabilizadas; se procedió a una extracción secuencial continua con Soxleth utilizando éter de petróleo, acetato de etilo, etanol, obteniéndose respectivamente 4 fracciones. Los extractos obtenidos fueron concentrados a presión reducida en un rotavapor.

\section{Aislamiento y purificación ${ }^{4,5}$}

Para el aislamiento y purificación se utilizó métodos de cromatografía en capa fina, cromatografía en columna, cromatografía líquida de alta eficacia y cristalización. Se trabajó con el extracto de acetato de etilo (mediana polaridad) con la finalidad de aislar y purificar productos medianamente polares de Ambrosia arborescens Miller.

El producto obtenido fue purificado por cromatografía en columna gravitatoria y de mediana presión. Se procedió a técnicas de cristalización para conseguir mayor grado de pureza. Para evaluar la pureza se recurrió a la cromatografía en capa fina (CCF o TLC). La determinación cuantitativa de la pureza se realizó con cromatografía líquida de alta efíciencia (HPLC). El extracto crudo de acetato de etilo se impregnó con silicagel para formar una cabeza de columna cromatográfica, lo que se colocó sobre una columna de silicagel 60, previamente empacada. Como fase móvil se empleó un sistema de solventes de polaridad creciente, partiendo de éter de petróleo, luego mezclas de éter de petróleo: acetato de etilo en proporciones de 9:1, 8:2, 7:3, etc., hasta llegar al 100\% de acetato de etilo. Por cada sistema se utilizó $100 \mathrm{~mL}$ de solventes. Los eluatos fueron recibidos en lotes de 25 tubos de ensayo.

Como resultado de estos procesos se obtuvo un producto sólido blanco al que se denominó Marco-1.

\section{Caracterización estructural ${ }^{4,6,7}$}

El producto purificado, fue estudiado a través de métodos espectroscópicos: Resonancia magnética nuclear protónica (RMN-1H), del carbono-13 (RMN-13C), espectrometría de masas (EM) y espectroscopía infrarroja (IR). Para establecer la estereoquímica se empleó técnicas espectroscópicas bidimensionales (COSY, ROESY, HMQC y HMBC).

\section{Análisis conformacional ${ }^{8}$}

Este trabajo fue apoyado con estudios teóricos de Mecánica Molecular usando el programa Hyperchem.

\section{Evaluación de actividad biológica}

Se realizó ensayo "in vitro" de inhibición del crecimiento de epimastigotes de cultivo. El compuesto obtenido se utilizó como posible inhibidor del crecimiento de parásitos. ${ }^{9,10,11}$

\section{RESULTADOS Y DISCUSIÓN}

Se ha obtenido un producto puro blanco cristalino con las siguientes características físicas: Punto de fusión: $214-216^{\circ} \mathrm{C}$; muestra un $\mathrm{Rf}=0,690$ (4:1:3:2 acetato de etilo: éter etílico: etanol: metanol); insoluble en agua, soluble en alcohol a altas temperaturas, de sabor amargo. El espectro de masas (EM) (figura 1), muestra un ion molecular, $\mathrm{M}^{+} 280,1490$ y un fragmento a m/z 262,1393 que correspondería a la pérdida de una molécula de agua $\left(\mathrm{M}^{+}-\mathrm{H}_{2} \mathrm{O}\right)$, lo que nos indica la presencia de un grupo hidroxilo. Un fragmento de masa m/z 247,1091 que correspondería a la pérdida consecutiva de agua y un grupo metilo $\left(\mathrm{M}^{+}-\mathrm{H}_{2} \mathrm{O}-\mathrm{CH} 3\right)$. También 
se ve un fragmento $\mathrm{m} / \mathrm{z} 237,1178$ característico de la pérdida de un ion acilio $\left(\mathrm{CH}_{3} \mathrm{C} \Xi \mathrm{O}^{+}\right)$. Esto es indicativo que un grupo metilo está unido al carbono portador de la función oxigenada (hidroxilo). El pico base m/z 125,035 corresponde a al fragmento $\mathrm{C}_{6} \mathrm{H}_{11} \mathrm{O}_{2}$.

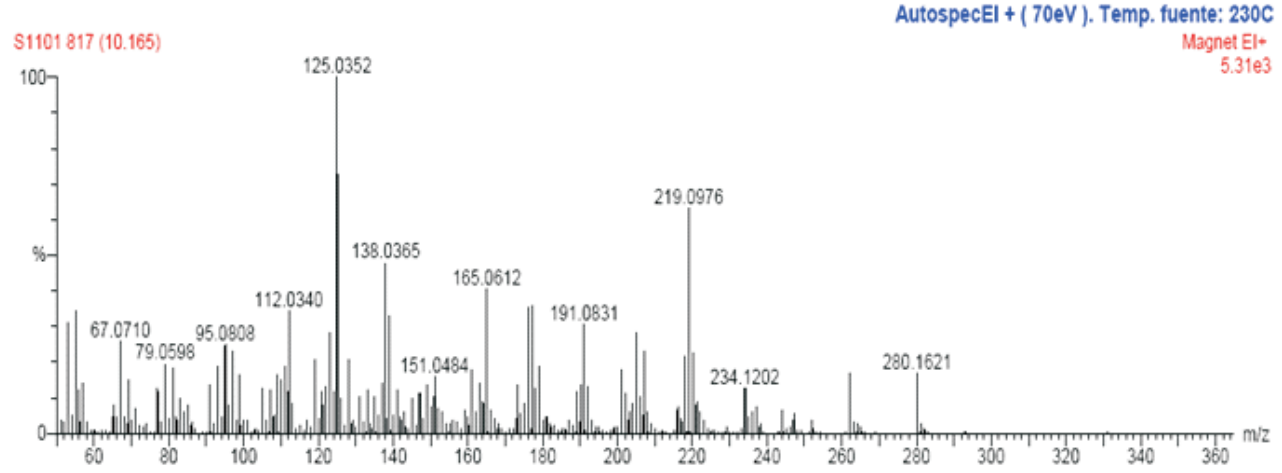

Figura 1. Espectro de masas de Marco-1

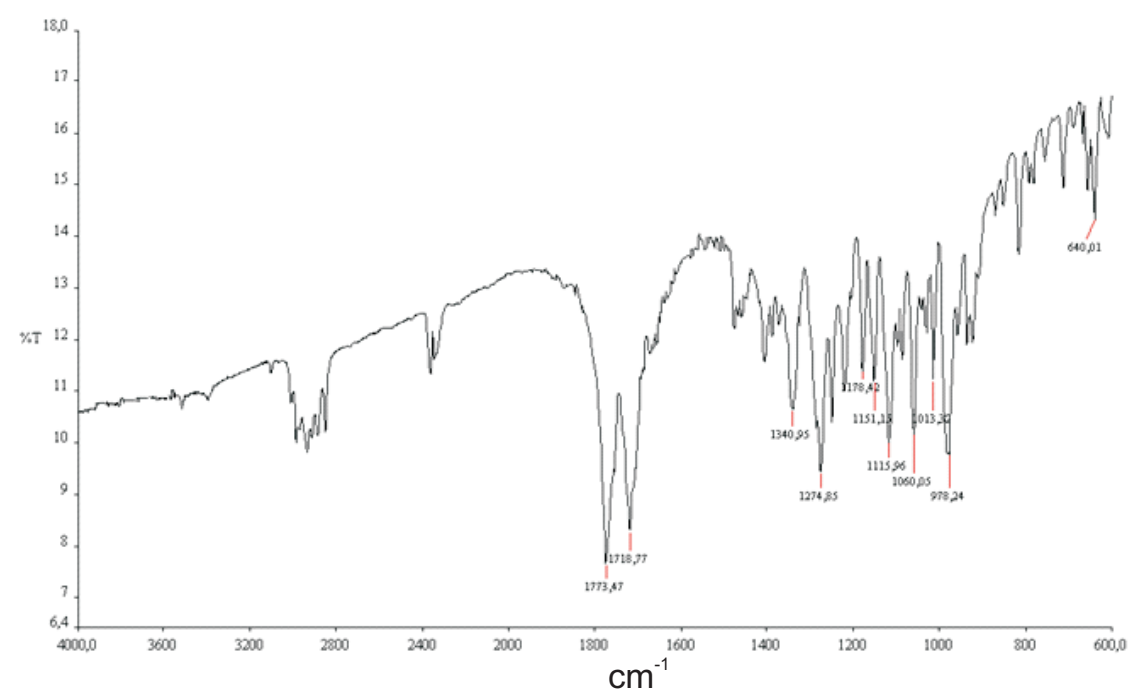

Figura 2. Espectro infrarrojo de Marco-1

En el espectro infrarrojo (figura 2) se observa claramente la presencia de 2 grupos carbonilo de la $\gamma$-lactona $\alpha$-metilénica a $1773 \mathrm{~cm}^{-1}$ y a $1718 \mathrm{~cm}^{-1}$, el carbonilo de la espirolactona.

Junto con lo anterior, el análisis del espectro de resonancia magnética nuclear protónica (RMN-1H), nos reafirma que estamos en presencia de una lactona sesquiterpénica $\alpha-$ metilénica $^{12}$ (figura 5). 
Cuando comparamos los datos espectroscópicos de $\mathrm{RMN}-1 \mathrm{H}$ con productos aislados de plantas del género Ambrosia, tales como: Psilostachina A I, Canambrina II y Cordilina III, (figura 3) se puede observar la similitud de los desplazamientos químicos como se puede observar en la tabla $1 .^{12,13}$

Psilostachiina A, I<smiles>C=C1C(=O)O[C@H]2[C@@H]1CC[C@H](C)[C@@]1(CCC(=O)O1)[C@@]2(C)O</smiles>

Canambrina, II<smiles>C=C1C(=O)OC2C1CCC(C)C1(CCC(=O)O1)[C@]2(C)O</smiles>

Cordilina, III

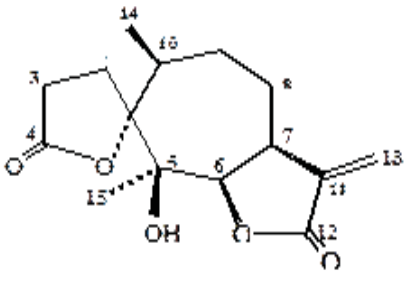

Figura 3. Estructuras de espirolactonas

Tabla 1. Comparación de los desplazamientos químicos en RMN -1H.

\begin{tabular}{ccccccc}
\hline COMPUESTO & H15 & H14 & H6 & H7 & H13 & P.F., ${ }^{\circ} \mathbf{C}$ \\
\hline I & $1,23 \mathrm{~s}$ & $1,05 \mathrm{~d}$ & $4,98 \mathrm{~d}$ & $3,4 \mathrm{~m}$ & $\begin{array}{l}5,53 \mathrm{~d} \\
6,29 \mathrm{~d}\end{array}$ & 215 \\
\hline II & $1,28 \mathrm{~s}$ & $1,20 \mathrm{~d}$ & $4,73 \mathrm{~d}$ & - & $\begin{array}{l}5,58 \mathrm{~d} \\
6,28 \mathrm{~d}\end{array}$ & $209-210$ \\
\hline III & $1,27 \mathrm{~s}$ & $1,19 \mathrm{~d}$ & $4,71 \mathrm{~d}$ & $3,33 \mathrm{~m}$ & $\begin{array}{l}5,75 \mathrm{~d} \\
6,29 \mathrm{~d}\end{array}$ & $210-211$ \\
\hline $\begin{array}{c}\text { Producto } \\
\text { obtenido }\end{array}$ & $1,32 \mathrm{~s}$ & $1,03 \mathrm{~d}$ & $4,67 \mathrm{~d}$ & $3,45 \mathrm{~m}$ & $\begin{array}{l}5,52 \mathrm{~d} \\
6,27 \mathrm{~d}\end{array}$ & $214-216$ \\
\hline
\end{tabular}

De los valores comparativos de desplazamiento químico de los protones más representativos: H6, H7, los protones metilénicos H13 y los protones de los grupos metilos H14 y H15, podemos hacer las asignaciones en el espectro de RMN-1H planteando como hipótesis de que se trata de un esqueleto con dos lactonas; una espirolactona y una $\alpha$-metilén- $\gamma$-butirolactona cis-fusionada, con un grupo hidroxilo y dos grupos metilos. Por tanto, quedaría por definir la estereoquímica de los centros quirales C1, C5, C6 y C7 y C10 como se muestra en las figuras 4 y 5 . 


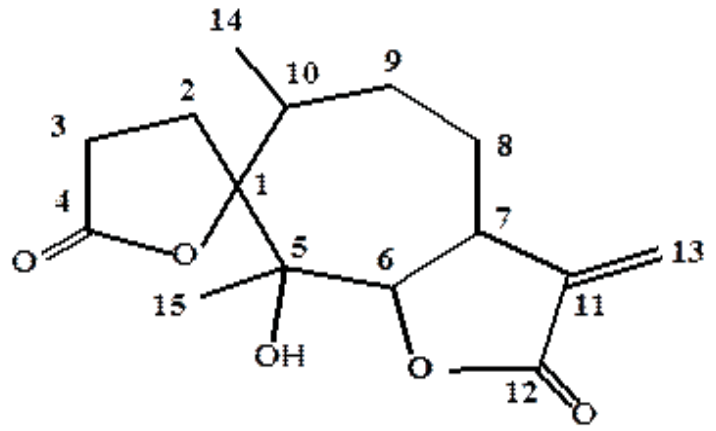

Figura 4. Esqueleto con dos lactonas; una espirolactona y una $\alpha$-metilen- $\gamma$-butirolactona cis-fusionada, con un grupo hidroxilo y dos grupos metilos.

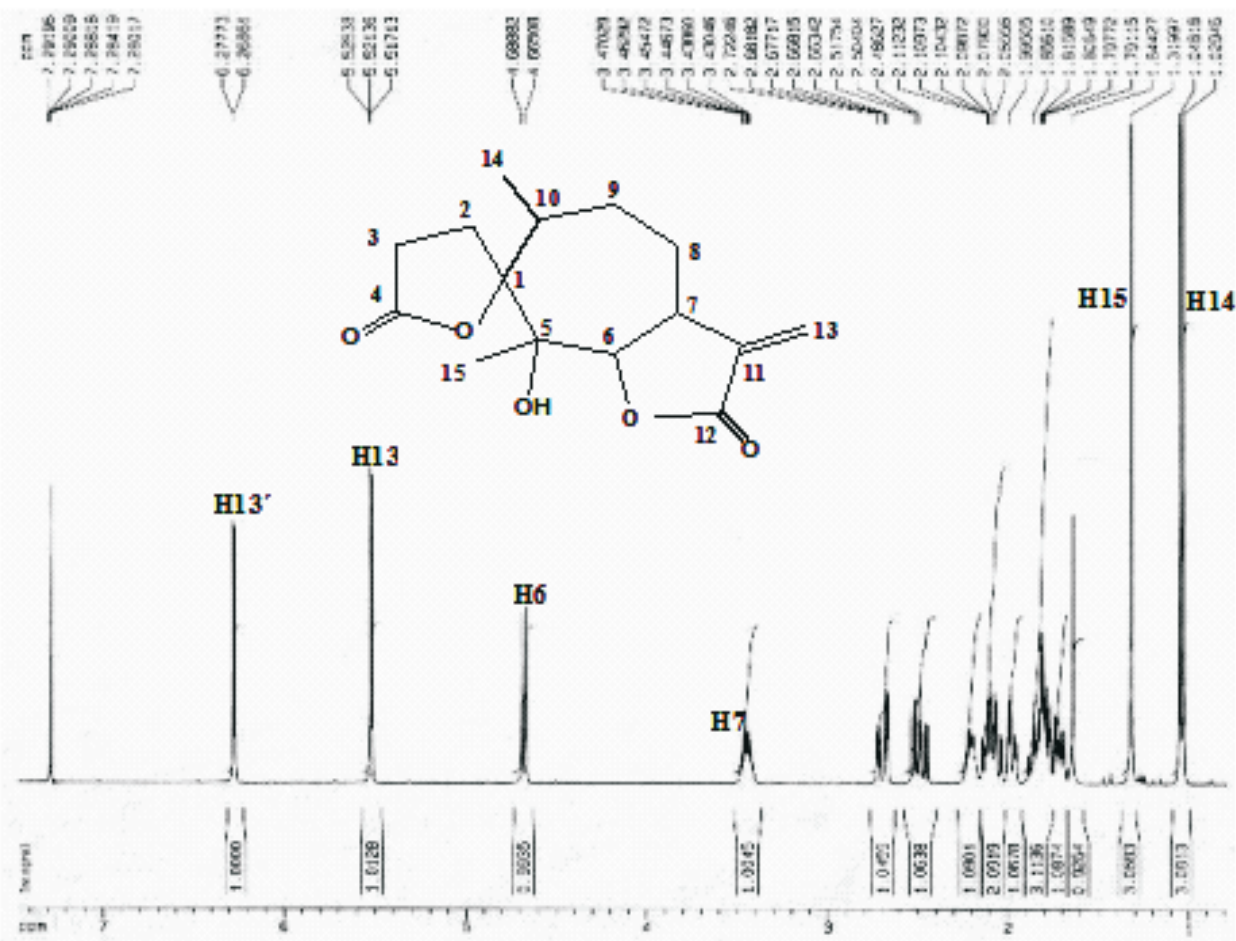

Figura 5. Espectro de resonancia magnética nuclear protónica del producto obtenido. 
El espectro de resonancia magnética nuclear del carbono-13 (RMN-13C) muestra 15 carbonos (figura 6), entre los cuales destacan dos carbonos carbonílicos en 169,97 y 169,31 ppm. Dos carbonos $\mathrm{sp}^{2}$ característicos de un metileno exocíclico a 138,81 y 120,78 ppm, respectivamente. Además, dos carbonos gemínales a funciones oxigenadas en 90,25 y 85,53 ppm.

Los desplazamientos químicos de los carbonos observados en el espectro son consistentes con la hipótesis planteada anteriormente y que señala la presencia de un sesquiterpeno con dos lactonas. Por otra parte, nos aporta evidencias de que el alcohol es terciario.

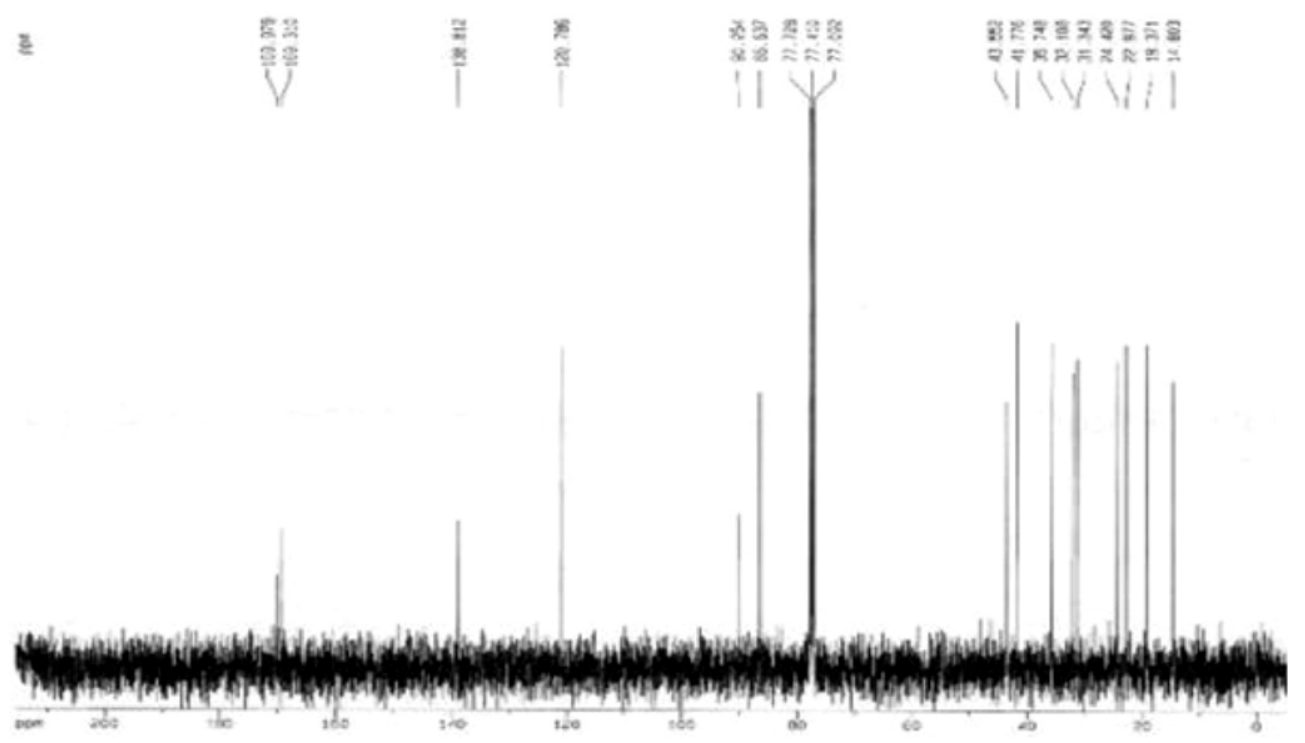

Figura 6. Espectro resonancia magnética nuclear de carbono-13 de Marco-1

Los valores de desplazamientos químicos y sus respectivas asignaciones se resumen en la tabla 2 . 
Tabla 2. Desplazamientos químicos de carbono-13 para Marco-1

\begin{tabular}{cccc}
\hline & $\begin{array}{c}\text { Desplazamientos químicos, } \\
\mathbf{p p m}\end{array}$ & Tipo de carbono & Asignación \\
1 & 169,978 & Carbonilo & $\mathrm{C} 4$ \\
2 & 169,310 & Carbonilo & $\mathrm{C} 12$ \\
3 & 138,812 & Cuaternario & $\mathrm{C} 11$ \\
4 & 120,786 & $\mathrm{CH} 2$ & $\mathrm{C} 13$ \\
5 & 90,254 & Cuaternario & $\mathrm{C} 1$ \\
6 & 86,637 & $\mathrm{CH}$ & $\mathrm{C} 6$ \\
7 & 43,552 & $\mathrm{Cuaternario}$ & $\mathrm{C} 5$ \\
8 & 41,776 & $\mathrm{CH}$ & $\mathrm{C} 7$ \\
9 & 35,748 & $\mathrm{CH}$ & $\mathrm{C} 10$ \\
10 & 32,108 & $\mathrm{CH} 2$ & $\mathrm{C} 3$ \\
11 & 31,343 & $\mathrm{CH} 2$ & $\mathrm{C} 9$ \\
12 & 24,420 & $\mathrm{CH} 2$ & $\mathrm{C} 2$ \\
13 & 22,977 & $\mathrm{CH} 2$ & $\mathrm{C} 8$ \\
14 & 19,371 & $\mathrm{CH} 3$ & $\mathrm{C} 15$ \\
15 & 14,803 & $\mathrm{CH} 3$ & $\mathrm{C} 14$ \\
\hline
\end{tabular}

Para determinar la estereoquímica de los centros quirales del producto obtenido, se analiza el espectro bidimensional COSY (Correlation Spectrsocopy) para detectar las correlaciones de los protones syn-orientados. 


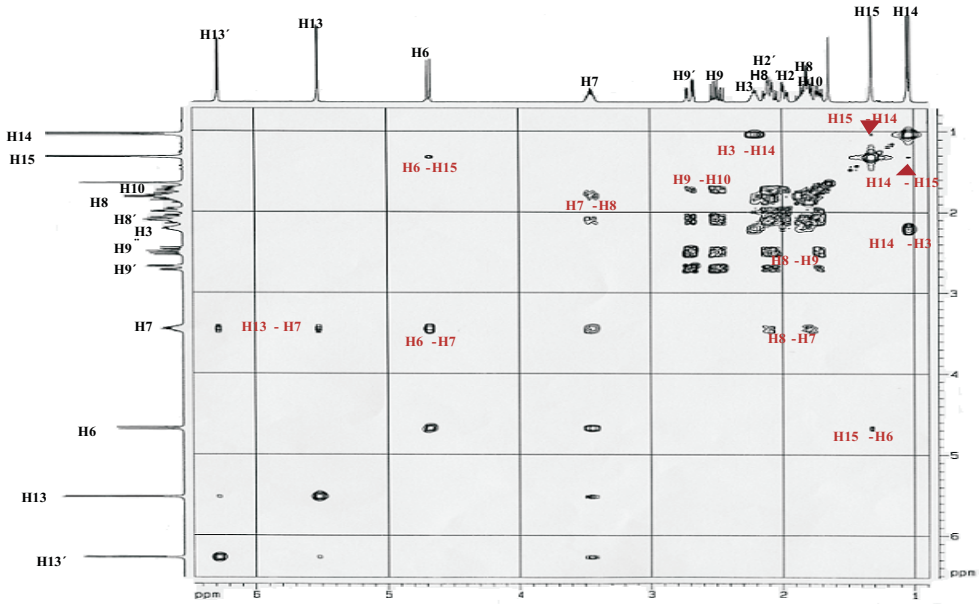

Figura 7. Espectro COSY de Marco-1

El espectro COSY, (figura 7) nos muestra el acoplamiento entre los protones $\mathrm{H}_{6}-\mathrm{H}_{7}$ lo que significa claramente que se trata de una lactona cis-fusionada. La interacción entre $\mathrm{H}_{3}-\mathrm{H}_{14}$ nos da indicación que la espirolactona tiene una configuración alfa. Es decir, el oxígeno lactónico tendría una disposición anti con el metilo sobre $\mathrm{C}_{10}\left(\mathrm{H}_{14}\right)$. Por otra parte, la interacción $\mathrm{H}_{6}-\mathrm{H}_{15}$ $\left(\mathrm{C}_{5} \mathrm{Me}\right)$ indica una syn orientación entre ambos. Este hecho es de particular importancia ya que descarta el hecho de que Marco-1 pudiera tener la estructura de Psilostachiina A y Canambrina, puesto que ambas tienen una disposición anti entre H6 y H15. Por otra parte, la correlación, aunque débil, H14 y H15, descarta la estructura de Cordilina. El acoplamiento pequeño entre estos protones correspondientes a los dos metilos, se puede explicar por la distancia entre ellos o, a que pudieran estar ecuatoriales, al menos uno de ellos.

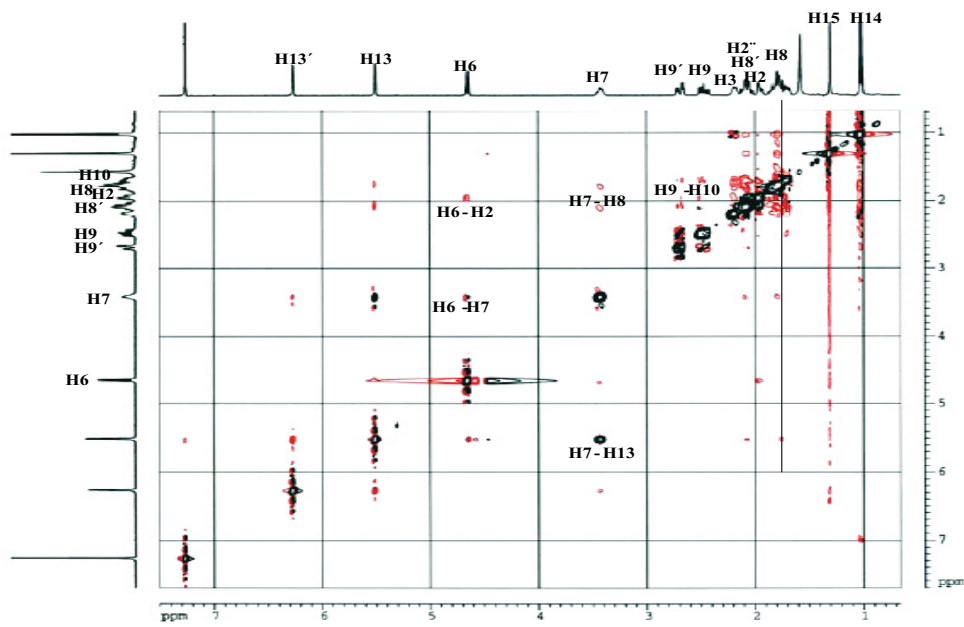

Figura 8. Espectro ROESY 
El espectro ROESY, (figura 8) (Rotational nuclear Overhauser Effect Spectroscopy), técnica que muestra los protones que están próximos en el espacio pero no cercanamente unidos a través de enlaces químicos.

El espectro ROESY es concordante con el espectro COSY. Se puede destacar que también se pueden ver las interacciones de los protones H6-H7 que confirman la fusión cis de la lactona $\alpha$-metilénica. Se debe resaltar que en el ROESY se observa la proximidad de H2, de la espirolactona, con el protón H6 del cierre lactónico, lo cual no era detectado en el espectro COSY. Esto confirma que la espirolactona tiene su oxígeno dispuesto anti respecto a H6.

En resumen, los espectros bidimensionales nos indican que los protones H6, H7, H14 y H15 están syn orientados. Por tanto, si asumimos que biogenéticamente H14 debería tener una orientación beta.

Las interacciones observadas en el experimento ROESY se pueden resumir en la siguiente figura 9:

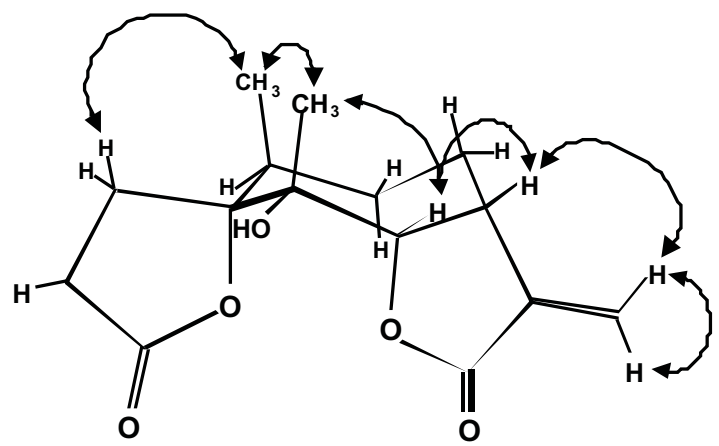

Figura 9. Experimento ROESY de Marco-1

Junto con establecer la estereoquímica de todos los centros quirales de la molécula, se puede observar que en solución, Marco-1, adopta una conformación CT (chair-twist chair).

Los datos espectroscópicos analizados nos permiten proponer para Marco-1 una estructura de sesquiterpeno con dos lactonas, una espirolactona y una $\alpha$-metilén- $\gamma$-butirolactona cisfusionada en alfa, cuya estructura se presenta a continuación (figura 10).

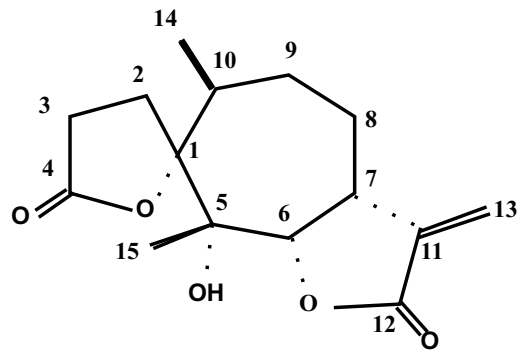

Figura 10. Estructura del producto obtenido 
Basándonos en los antecedentes bibliográficos, es una molécula nueva. Por tanto, proponemos para esta molécula el nombre de "Ambrosina A", (1R, 5S, 6 $\alpha \mathrm{R}, 7 \alpha \mathrm{S}, 10 \mathrm{~S})$ 5hidroxi-5,10-dimetil-11-metilén-1-oxospiro- [4,6]-undecan-4-oxa-6,12-olida.

\section{Análisis conformacional}

Para establecer la conformación de las lactonas sesquiterpénicas, se usó la mecánica molecular, técnica que basándose en la mecánica clásica, trata de evaluar el contenido energético y la disposición espacial de las moléculas orgánicas.

El análisis conformacional fue realizado a través de mecánica molecular empleando el programa MM2 de Hyperchem ${ }^{8}$. Los cálculos de las superficies de potencial representan al movimiento de los enlaces C1-C5-C6 y C8-C9-C10 del anillo B de la molécula ya que, los demás enlaces no presentan movilidad conformacional.

Estos cálculos muestran que el producto obtenido puede presentar cuatro conformaciones: CC: $38,38 \mathrm{kcal} / \mathrm{mol}, \mathrm{CT}: 43,61 \mathrm{kcal} / \mathrm{mol}$, TT: 47,50 kcal/mol, TT: 49,97 kcal/mol.

La conformación estable presenta menor energía (figura 11).

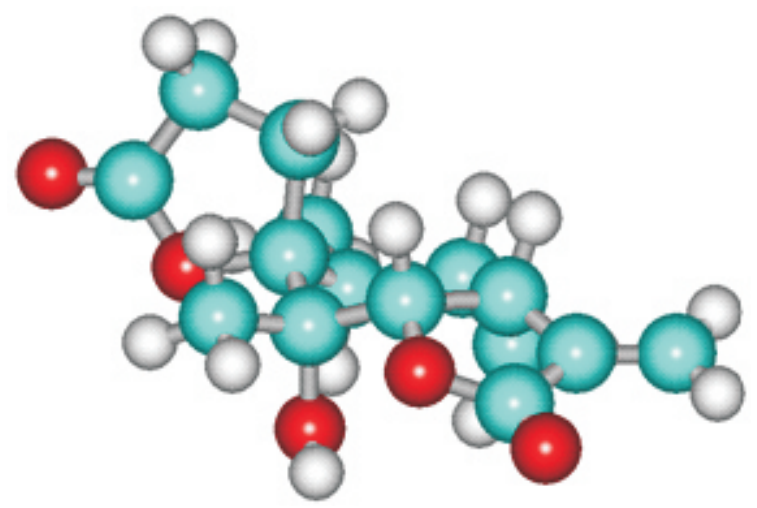

Figura 11. Energía conformacional, $38,38 \mathrm{kcal} / \mathrm{mol}$.

\section{Evaluación de la actividad biológica}

Para evaluar la actividad tripanocida de los productos se realizó ensayo in vitro de inhibición del crecimiento de epimastigotes de cultivo. Los compuestos obtenidos se utilizaron como posibles inhibidores del crecimiento de parásitos. Los epimastigotes de la cepa Tulahuén, stock Tul 2, fueron cultivados en presencia de diferentes concentraciones de las drogas. La inhibición del crecimiento parasitario en relación a los controles fue evaluado. Los resultados obtenidos indicaron que los productos aislados tienen actividad tripanocida, lo que se reporta en la tabla 3.

Tabla 3. Actividad tripanocida de los productos obtenidos de Ambrosia arborescens.

$\begin{aligned} & \text { Producto } \\ & \text { obtenido }\end{aligned} \quad 10 \mathrm{microg} / \mathrm{ml} \quad$ Etanol caliente (Mala)




\section{CONCLUSIONES}

- Mediante los estudios químicos espectroscópicos y conformacionales se ha podido establecer que el producto obtenido es una estructura nueva al que le corresponde el nombre IUPAC de (1R, 5S, 6 $\alpha \mathrm{R}, 7 \alpha \mathrm{S}, 10 \mathrm{~S})$-5-hidroxi-5,10-dimetil-11-metilén-1oxospiro-[4,6]-undecan-4-oxa-6,12-olida. La estructura que se ha determinado corresponde a una lactona sesquiterpénica con dos anillos lactónicos. Un anillo espirolactónico y otro $\alpha$-metilén- $\gamma$-butirolactona.

- El estudio teórico a través de mecánica molecular muestra que "Ambrosina A" puede presentar cuatro conformaciones posibles centradas en el anillo cicloheptánico: CC, CT, TT y TT, siendo la conformación más estable $\mathrm{CC}$, cuya energía conformacional es de $38.38 \mathrm{kcal} / \mathrm{mol}$.

- $\quad$ Los experimentos in vitro de la actividad tripanocida mostraron a bajas concentraciones. El producto obtenido presenta una actividad importante.

\section{AGRADECIMIENTO}

Agradezco al equipo de investigación del Proyecto "CHAGASPACE ", de la Universidad Católica del Norte, Antofagasta-Chile.

\section{BIBLIOGRAFÍA}

1. Rodríguez, M. "Estudio de la Biodiversidad Cuenca del Cotahuasi”, Editorial AEDES, La Unión-Arequipa, 1998

2. Sotta, N. Plantas Aromáticas y Medicinales de la Región Arequipa. Editorial Akuarella. Arequipa-Perú 2000

3. Palacios, J. Plantas Medicinales Nativas del Perú. Consejo Nacional de Ciencia y Tecnología Ed. CONCYTEC. Lima-Perú. 1997

4. Cano, Teresa; Extracción, separación, caracterización y evaluación de la actividad tripanocida de los metabolitos secundarios obtenidos de Ambrosia arborescens Miller; (Tesis de Doctorado) UNSA-Arequipa Perú 2005

5. Golsby, G. y Burke, A. Sesquiterpenlactonas y sesquiterpenodiol de Ambrosia peruviana de Jamaica. Phytochemistry. 1987; 26 (4): 1059-1063

6. Hesse, M.; Meier, H. and Zeeh, B. Métodos Espectroscópicos en Química Orgánica, Editorial Síntesis, Madrid-España, 2005

7. Martínez, A. Sesquiterpenlactonas. Universidad de Antioquia, Medellín-Colombia, 2002.

8. Hyperchem Release 7 Tools for Molecular Modeling, USA, 2002.

9. Fichera, L.; Esteva, M.; Wimmer, Z.; Rodríguez, JB.; and Gros, E. Effects of juvenile hormone analogues (JHA) on the development of Tripanosoma cruzi. Zeitschrift fur Naturforsh, Journal of biosciences, 1995; 50 (7): 578-580

10. Otoguro, K.; Iwatsuki, M.; Ishiyama, A.; Namatame, M.; Nishihara-Tukashima, A.;Kiyohara, H.; Hashimoto, T.; Asakawa, Y.; Omura, S. and Yamada , H. In vitro antitrypanosomal activity of plant terpenes against Trypanosoma brucei. Fitoterapia. 2011;72 (4): 2024-2030

11. Araya, J.; Neira, I.; da Silva, S.; Mortara R.; Manque P.; Cordero E.; Sagua H.; Loyola A.; Bórquez J.; Morales G.; Gonzáles J. Diterpenoids from Azorella compacta (Umbelliferae) Active on Trypanosoma cruzi. Mem Inst Oswaldo Cruz.2003;98 (3): 413-418

12. Herz, W.; Rau, D. and Anderson, D. Sesquiterpene Lactonas of Ambrosia cordifolia. Phytochemistry, 1973; $12(6): 1415-1420$

13. Romo de Vivar A. Productos Naturales de la Flora Mexicana, Editorial LIMUSA, Mexico, 1985 